\title{
Quantum Fluctuations of Charge Order Induce Phonon Softening in a Superconducting Cuprate
}

\author{
H. Y. Huang $\odot,{ }^{1}$ A. Singh $\odot,{ }^{1}$ C. Y. Mou $\odot,{ }^{2}$ S. Johnston, ${ }^{3}$ A. F. Kemper $\odot,{ }^{4}$ J. van den Brink, ${ }^{5}$ P. J. Chen, ${ }^{6,7}$ T. K. Lee $\odot,{ }^{8,9}$ \\ J. Okamoto®, ${ }^{1}$ Y. Y. Chu, ${ }^{1}$ J. H. Li, ${ }^{10,1}$ S. Komiya, ${ }^{11}$ A. C. Komarek, ${ }^{12}$ A. Fujimori $\odot,{ }^{13,1}$ C. T. Chen $\odot,{ }^{1}$ and D. J. Huang ${ }^{1,10, *}$ \\ ${ }^{1}$ National Synchrotron Radiation Research Center, Hsinchu 30076, Taiwan \\ ${ }^{2}$ Center for Quantum Technology and Department of Physics, \\ National Tsing Hua University, Hsinchu 30013, Taiwan \\ ${ }^{3}$ Department of Physics and Astronomy, The University of Tennessee, Knoxville, Tennessee 37996, USA \\ ${ }^{4}$ Department of Physics, North Carolina State University, Raleigh, North Carolina 27695, USA \\ ${ }^{5}$ Institute for Theoretical Solid State Physics, IFW Dresden, \\ Helmholtzstrasse 20, D-01069 Dresden, Germany \\ ${ }^{6}$ Department of Mechanical Engineering, City University of Hong Kong, \\ Kowloon, Hong Kong 999077, China \\ ${ }^{7}$ Hong Kong Institute for Advanced Study, City University of Hong Kong, \\ Kowloon, Hong Kong 999077, China \\ ${ }^{8}$ Institute of Physics, Academia Sinica, Taipei 11529, Taiwan \\ ${ }^{9}$ Department of Physics, National Sun Yat-sen University, Kaohsiung 80424, Taiwan \\ ${ }^{10}$ Department of Physics, National Tsing Hua University, Hsinchu 30013, Taiwan \\ ${ }^{11}$ Central Research Institute of Electric Power Industry, Yokosuka, Kanagawa 240-0196, Japan \\ ${ }^{12}$ Max Planck Institute for Chemical Physics of Solids, Nöthnitzer Straße 40, 01187 Dresden, Germany \\ ${ }^{13}$ Department of Applied Physics, Waseda University, Shinjuku-ku, Tokyo 169-8555, Japan
}

(Received 3 April 2021; revised 13 August 2021; accepted 29 September 2021; published 23 November 2021)

\begin{abstract}
Quantum phase transitions play an important role in shaping the phase diagram of high-temperature cuprate superconductors. These cuprates possess intertwined orders which interact strongly with superconductivity. However, the evidence for the quantum critical point associated with the charge order in the superconducting phase remains elusive. Here, we reveal the short-range charge orders and the spectral signature of the quantum fluctuations in $\mathrm{La}_{2-x} \mathrm{Sr}_{x} \mathrm{CuO}_{4}$ (LSCO) near the optimal doping using highresolution resonant inelastic $\mathrm{x}$-ray scattering. On performing calculations through a diagrammatic framework, we discover that the charge correlations significantly soften several branches of phonons. These results elucidate the role of charge order in the LSCO compound, providing evidence for quantum critical scaling and discommensurations associated with charge order.
\end{abstract}

DOI: $10.1103 /$ PhysRevX.11.041038

\section{INTRODUCTION}

When doped with holes or electrons, cuprates at low temperatures can be tuned from a Mott insulating phase to a superconducting phase and then to a Fermi-liquid phase. In addition, an enigmatic pseudogap phase exists in the underdoped regime of hole-doped cuprates above the superconductivity transition temperature $T_{C}$ with a crossover temperature $T^{*}$, which decreases monotonically when the doping is increased [1]. Several symmetry-breaking orders such as charge-density waves (CDWs) have also

*djhuang@nsrrc.org.tw

Published by the American Physical Society under the terms of the Creative Commons Attribution 4.0 International license. Further distribution of this work must maintain attribution to the author(s) and the published article's title, journal citation, and DOI.

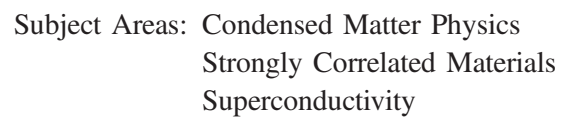

been discovered in the cuprates with comparable onset temperatures [2].

Charge fluctuations are one of the most fundamental collective excitations in matter. Recently, CDWs in cuprate superconductors have attracted renewed interest [3-25], but their mechanism and competition with superconductivity remain subjects of vigorous discussion. The wave vector of the CDW, for example, exhibits an inconsistent evolution with doping in various cuprate families $[7,8]$. This observation has lead to debate on whether real-space local interactions or Fermi surface nesting in momentum space underlies the CDW physics. Moreover, CDWs are coupled to phonons in cuprates [22-33], but the underlying mechanism of phonon softening remains a mystery [6], although lattice vibrations in metals can be damped or softened by electronic quasiparticles or excitations. 
Upon tuning a nonthermal parameter through a critical value, quantum phase transitions occur at the absolute zero of temperature $T$. The putative quantum critical point (QCP) in a cuprate holds the key to understanding many profound phenomena related to its superconductivity [34-40]. Anomalous thermodynamic [38] and transport properties $[39,40]$ of cuprates close to a QCP have been observed. For example, as temperature $T$ approaches zero, the electrical resistivity varies linearly with $T$ rather than the $T^{2}$ dependence of a Fermi liquid. In approaching a QCP, two quantum states of the system can exchange their energy ordering. As a result, a CDW state can be intertwined with the superconducting state. Also, because the energy difference is small, switching back and forth between CDW and superconducting ground states is energetically likely, resulting in quantum charge fluctuations. Interestingly, recent resonant inelastic $\mathrm{x}$-ray scattering (RIXS) studies have found that the charge-density fluctuations permeate through a broad region of the cuprate phase diagram [10].

A crucial task in the scenario of the presence of QCP is to identify the ordered phases that terminates at the QCP. In the present work, we presume the ordered phases to be the CDW order and try to find evidence for the QCP associated with the CDW in the superconducting phase. Inelastic x-ray scattering (IXS) probes charge fluctuations by measuring the dynamical structure factor $S(\mathbf{q}, \omega)$, which is the space and time Fourier transformation of the density-density correlation function. Here, $\hbar \mathbf{q}$ and $\hbar \omega$ are the momentum and energy transferred to charge excitations, respectively, with $\hbar$ being the Planck constant $h$ divided by $2 \pi$. For a given electron system of charge density $n(\mathbf{r}, t)$ at position $\mathbf{r}$ and time $t$, the charge fluctuation is $\delta n(\mathbf{r}, t)=n(\mathbf{r}, t)-\langle n(\mathbf{r}, t)\rangle$, in which $\langle\cdots\rangle$ denotes the quantum statistical average. The densitydensity correlation function $\left\langle n(\mathbf{r}, t) n\left(\mathbf{r}^{\prime}, t^{\prime}\right)\right\rangle$ can then be written as

$\left\langle n(\mathbf{r}, t) n\left(\mathbf{r}^{\prime}, t^{\prime}\right)\right\rangle=\langle n(\mathbf{r}, t)\rangle\left\langle n\left(\mathbf{r}^{\prime}, t^{\prime}\right)\right\rangle+\left\langle\delta n(\mathbf{r}, t) \delta n\left(\mathbf{r}^{\prime}, t^{\prime}\right)\right\rangle$.

In the presence of disorder, an additional average over disorders must be taken over Eq. (1). X-ray scattering data, thus, comprise contributions of the static charge distribution $\langle n(\mathbf{r}, t)\rangle\left\langle n\left(\mathbf{r}^{\prime}, t^{\prime}\right)\right\rangle$ and its dynamical fluctuations $\left\langle\delta n(\mathbf{r}, t) \delta n\left(\mathbf{r}^{\prime}, t^{\prime}\right)\right\rangle$, corresponding to charge susceptibilities denoted by $\chi_{0}(\mathbf{q}, \omega)$ and $\chi(\mathbf{q}, \omega)$, respectively. In addition, through the fluctuation-dissipation theorem, $S(\mathbf{q}, \omega)$ is related to the charge susceptibility $\chi(\mathbf{q}, \omega)$ by $S(\mathbf{q}, \omega)=2 \hbar\left(1-e^{-\beta \hbar \omega}\right)^{-1} \operatorname{Im} \chi(\mathbf{q}, \omega)$, in which $\beta=1 / k_{B} T$, with $k_{B}$ denoting the Boltzmann constant.

$\mathrm{O} K$-edge RIXS probes certain characteristics of the dynamical charge fluctuations of CDWs, although additionally modulated by the effects of RIXS matrix elements, light polarization, and orbital characters $[10,23,41,42]$. In this work, we perform high-resolution $\mathrm{O} K$-edge RIXS measurements and theoretical calculations through a diagrammatic framework to investigate the quantum fluctuations of charge order in superconducting cuprate $\mathrm{La}_{2-x} \mathrm{Sr}_{x} \mathrm{CuO}_{4}$ (LSCO) near the optimal doping. Our results elucidate the role of charge order in the LSCO compound, providing evidence for the quantum critical scaling associated with charge order.

\section{RIXS MEASUREMENTS}

\section{A. Experimental}

The LSCO single crystals with the doping level $x=0.15$ are grown by the traveling-solvent floating zone method [43-45]. After growth, the crystals are annealed to remove oxygen defects. The value of $x$ is determined from an inductively-coupled-plasma atomic-emission spectrometric analysis. The $T_{C}$ of the $x=0.15$ sample is $37.5 \mathrm{~K}$.

We conduct $\mathrm{O} K$-edge RIXS measurements using the AGM-AGS spectrometer of beam line 41A at Taiwan Photon Source of National Synchrotron Radiation Research Center, Taiwan [46]. This recently constructed AGM-AGS beam line is based on the energy compensation principle of grating dispersion. High-resolution RIXS data are measured with an energy resolution of $16 \mathrm{meV}$ at an incident photon energy of $530 \mathrm{eV}$. See Supplemental Material [47] for the details of the AGM-AGS spectrometer and data analysis.

\section{B. Charge order of LSCO}

We first measure static charge distributions $\langle n(\mathbf{r}, t)\rangle\left\langle n\left(\mathbf{r}^{\prime}, t^{\prime}\right)\right\rangle$ of LSCO with $x=0.15$ using energyresolved elastic x-ray scattering. Figure 1(a) plots the intensity of $\mathrm{O} K$-edge elastic scattering as a function of in-plane wave vector change $\mathbf{q}_{\|}$varied along the antinodal direction $(\pi, 0)$ at various temperatures. We observe CDW correlations in LSCO with an in-plane modulation vector $\mathbf{q}_{\mathrm{CDW}}=(0.235,0)$, which is given in reciprocal lattice units (r.l.u.) throughout this paper. Figures 1(c) and 1(d) show, respectively, the temperature-dependent CDW intensity and correlation length $(\xi)$ defined as the inverse of the half width at half maximum (HWHM) of the momentum scan. The charge order of optimally doped LSCO exhibits a short correlation length, ranging from 22 to $12 \AA$. Consistent with previous results of LSCO, the CDWs are slightly suppressed in the superconducting phase as the temperature is decreased across $T_{C}[5,9]$.

The observed charge correlations of LSCO persist over a wide temperature range, up to well above $T^{*}$ and into the strange-metal phase. These correlations achieve no longrange charge order, however, in contrast with other La-214 cuprates. For example, $\mathrm{La}_{1.48} \mathrm{Nd}_{0.4} \mathrm{Sr}_{0.12} \mathrm{CuO}_{4}$ (Nd-LSCO), which has a low-temperature tetragonal (LTT) structure, shows long-range and short-range charge orders, as plotted in Fig. 1(b). The short-range charge order of LSCO might be caused by spatial disorders or phase separation arising 

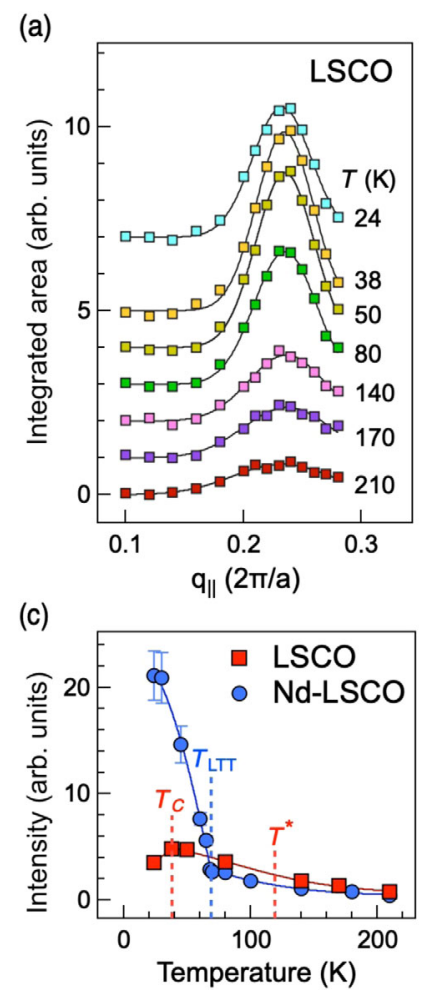

intensity that has a maximum at $T_{C}$ as plotted in Fig. 1(c) with red squares. This low-energy RIXS excitation appears to shift away from $\mathbf{q}_{\mathrm{CDW}}$ when the thermal energy approaches the excitation energy. This reflects that, at low temperatures, the low-energy RIXS excitation is dominated by charge-density fluctuations and its contribution from the coupling of CDW to acoustic phonons increases with the increase of temperature, as discussed later.

\section{CDW fluctuations}

For a system that exhibits a QCP associated with charge order, $S(\mathbf{q}, \omega)$ near $\mathbf{q}_{\mathrm{CDW}}$ measures the collective fluctuations sensitive to the proximity of the QCP. When the thermal energy $k_{B} T$ is less than the typical energy of order parameter fluctuations and less than energy $\hbar \omega$, their quantum nature is important. For low temperatures, we expect that the finite frequency fluctuation is dominated by the amplitude fluctuation of the charge density, and the following phenomenological form for charge susceptibility describes the quantum fluctuations $[10,35]$ :

$$
\chi_{\mathrm{CDW}}\left(\mathbf{q}_{\|}, \omega\right)=\frac{1}{\Delta^{2}+c^{2}\left(\mathbf{q}_{\|}-\mathbf{q}_{\mathrm{CDW}}\right)^{2}-\left(\omega+i \Gamma_{\mathrm{CDW}}\right)^{2}} .
$$

Scattering intensities of LSCO and Nd-LSCO vs in-plane momentum $\mathbf{q}_{\|}$along the antinodal direction $(\pi, 0)$ at various temperatures. Energy-resolved elastic-scattering plots are extracted from the integrated area of $\mathrm{O} K$-edge RIXS over the energy from -5 to $5 \mathrm{meV}$. The incident $\mathrm{x}$-ray energy for the RIXS spectra is tuned to the mobile hole of the so-called Zhang-Rice singlet (ZRS) with an absorption energy near $528.5 \mathrm{eV}$. All spectra are vertically offset for clarity. (c),(d) CDW intensity and correlation length $(\xi)$ of LSCO and Nd-LSCO as a function of the temperature, respectively. The vertical dashed lines show $T_{C}$ and $T^{*}$ of LSCO and structural transition temperature $T_{\text {LTT }}$ of Nd-LSCO. All solid lines serve as visual guidance.

from a subtle balance between Coulomb interactions and kinetic energy. At this time, we cannot determine if the CDWs with $\mathbf{q}_{\mathrm{CDW}}=(0.235,0)$ are truly incommensurate or instead appear incommensurate due to defects known as discommensurations in the commensurate CDW state [48-51]. In a discommensurated structure, locally commensurate CDW regions are separated by discommensurations within which the CDW phase varies rapidly.

To unveil the charge instability and the coupling between CDW fluctuations and phonons in LSCO, we perform high-resolution O $K$-edge RIXS measurements. Figure 2(a) presents a series of temperature-dependent RIXS intensity distribution maps in the plane of energy loss vs in-plane momentum transfer $\mathbf{q}_{\|}$along ( $\left.\pi, 0\right)$; Fig. 2(b) shows those maps after the subtraction of elastic scattering. There exists a pronounced excitation feature of energy about $14 \mathrm{meV}$ in the neighborhood of $\mathbf{q}_{\mathrm{CDW}}$. Its intensity decreases with increasing temperature, unlike that of the static CDW

Here, $\Delta$ is the characteristic energy of the fluctuations at $T=0$ and can be interpreted as the inverse correlation length for the amplitude fluctuations. One expects that the characteristic energy $\Delta$ vanishes when the doping level approaches the quantum critical point. $c$ is a parameter that characterizes the speed of the excitations with dispersion $\omega \sim c\left|\mathbf{q}_{\|}-\mathbf{q}_{\mathrm{CDW}}\right|$, and $\Gamma_{\mathrm{CDW}}$ is a parameter that characterizes the lifetime of the CDW amplitude excitations. In contrast, the static charge susceptibility $\chi_{0}(\mathbf{q}, \omega)$ vanishes except for $\omega \sim 0$ and its nearby region of CDW phase fluctuations characterized by Eq. (2) with $\Delta=0$ and parameters $c$ and $\Gamma_{\mathrm{CDW}}$. This energy region is beyond our RIXS energy resolution.

Figure 3(a) plots RIXS spectra after the subtraction of elastic scattering for momentum integrated from $q_{\|}=0.22$ to $q_{\|}=0.25$ at different temperatures. This low-energy RIXS intensity decreases when the temperature is increased as plotted in Fig. 3(b). On comparing the intensity, spectral profile, and peak energy of the low-energy RIXS excitation near $14 \mathrm{meV}$ with those of calculated $S\left(\mathbf{q}_{\mathrm{CDW}}, \omega\right)$, in which the contribution of the dispersion parameter $c$ vanishes, one can obtain the evolution of $\Delta$ and $\Gamma_{\mathrm{CDW}}$ for various temperatures. Figures 3(c) and 3(d) show the estimated temperature-dependent $\Delta$ and $\Gamma_{\mathrm{CDW}}$, respectively. The evolution of $\Delta$, which is inversely proportional to the CDW correlation length [36], is accordant with the temperature dependence of static charge order shown in Fig. 1. At low temperatures, $\Gamma_{\mathrm{CDW}}$ grows with increasing temperature. In the quantum critical regime, the scattering rate of 

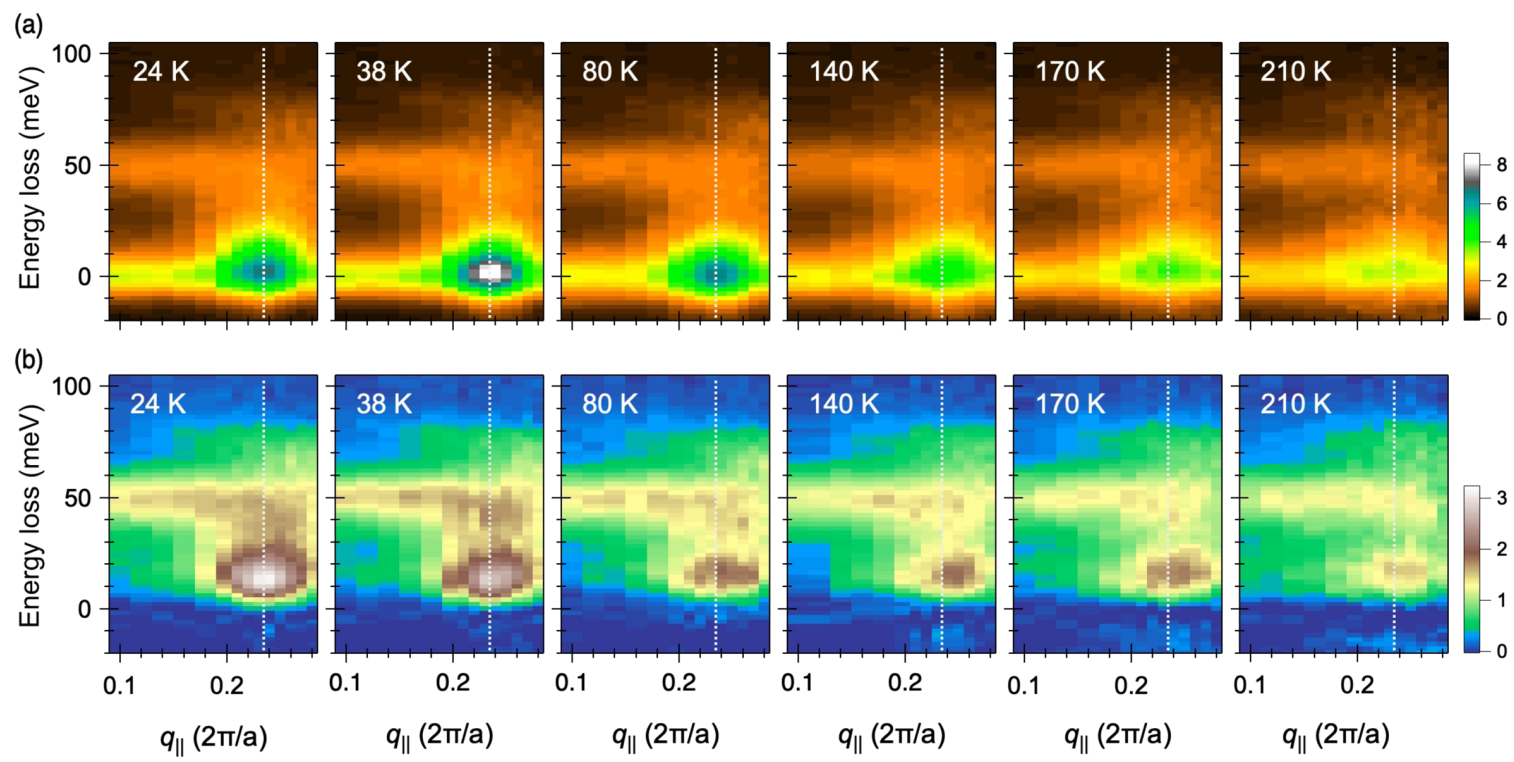

FIG. 2. Temperature-dependent O $K$-edge RIXS of optimally doped LSCO. (a) RIXS intensity distribution maps in the plane of energy loss vs in-plane momentum transfer $\mathbf{q}_{\|}$along $(\pi, 0)$ at various temperatures. (b) RIXS intensity distribution maps after the subtraction of elastic scattering. All RIXS spectra are recorded with $\sigma$-polarized incident x rays of energy tuned to the ZRS hole. The momentum transfer is $\mathbf{q}=\left(q_{\|}, 0, L\right)$ with $L$ varying between 0.47 and 1.03 in reciprocal lattice units. Dotted lines indicate the position of measured $\mathbf{q}_{\mathrm{CDW}}=(0.23,0, L)$.

quasiparticles reaches the Planckian limit, and the change of $\Gamma_{\mathrm{CDW}}$ is comparable with $k_{B} T / \hbar$, i.e., the Planckian dissipation $[40,52]$. Although the measured data cover only one temperature point below $T_{C}$, this observation supports the quantum critical nature of CDW fluctuations in LSCO.

Figure 3(d) also shows that, as $T$ is increased, the temperature dependence of $\Gamma_{\mathrm{CDW}}$ exhibits a bending feature about $80 \mathrm{~K}$. This suggests two possible scenarios: a crossover near the QCP and the coupling of CDW fluctuations to acoustic phonons. First, a crossover of $\Gamma_{\mathrm{CDW}}$ behavior near the QCP $[53,54]$ indicates that Planckian linear- $T$ dependence works only at the critical regime (low-temperature regions) controlled by the QCP. In other words, only inside the critical regime, $\Gamma_{\mathrm{CDW}}$ shows the linear- $T$ dependence, which reflects the gapless nature at the QCP, i.e., $1 / \Gamma_{\mathrm{CDW}}$ going to infinity. At higher temperatures beyond the critical regime, $\Gamma_{\mathrm{CDW}}$ no longer reaches the Planckian limit. In this scenario, the quantum critical region is up to approximately $80 \mathrm{~K}$. In the second scenario, the bending feature of $\Gamma_{\mathrm{CDW}}$ suggests that, for the thermal energy $k_{B} T$ comparable to the charge fluctuation energy, CDW fluctuations alone do not explain the RIXS data at high temperatures, as shown in supplemental Figs. S8(e)-S8(g) [47]. This indicates that the low-energy RIXS is also contributed by the coupling of CDW fluctuations to phonons. We, hence, calculate the full RIXS intensity using a diagrammatic framework, in which the coupling between $\chi_{\mathrm{CDW}}$ and phonons renormalizes the phonon propagator.

\section{RIXS CALCULATIONS}

The intensity of the phonon excitations is calculated by using the diagrammatic framework [55] generalized to the $\mathrm{O} K$ edge. The RIXS intensity is given by $(\hbar=1)$

$$
\begin{aligned}
I_{\nu}(\mathbf{q}, \Omega)= & \frac{1}{N} \sum_{\mathbf{k}, \alpha} \frac{1}{N} \sum_{\mathbf{k}^{\prime}, \beta}\left[\hat{\mathbf{\epsilon}}_{i} \cdot \hat{\mathbf{r}}_{\alpha}\right]\left[\hat{\mathbf{\epsilon}}_{f} \cdot \hat{\mathbf{r}}_{\beta}\right] g_{\nu}(\mathbf{k}, \mathbf{k}+\mathbf{q})\left|\phi_{2 p_{\alpha}}(\mathbf{k})\right|^{2}\left|\phi_{2 p_{\beta}}(\mathbf{k}+\mathbf{q})\right|^{2}\left[\frac{n_{f}\left(-\epsilon_{\mathbf{k}}\right)}{\epsilon_{\mathbf{k}}-\omega_{f}-E_{1 s, 2 p}-i \Gamma}-\frac{n_{f}\left(-\epsilon_{\mathbf{k}+\mathbf{q}}\right)}{\epsilon_{\mathbf{k}+\mathbf{q}}-\omega_{i}-E_{1 s, 2 p}-i \Gamma}\right] \\
& \times g_{\nu}\left(\mathbf{k}^{\prime}+\mathbf{q}, \mathbf{k}^{\prime}\right)\left|\phi_{2 p_{\beta}}\left(\mathbf{k}^{\prime}\right)\right|^{2}\left|\phi_{2 p_{\alpha}}\left(\mathbf{k}^{\prime}+\mathbf{q}\right)\right|^{2}\left[\frac{n_{f}\left(-\epsilon_{\mathbf{k}^{\prime}}\right)}{\epsilon_{\mathbf{k}^{\prime}}-\omega_{f}-E_{1 s, 2 p}+i \Gamma}-\frac{n_{f}\left(-\epsilon_{\mathbf{k}^{\prime}+\mathbf{q}}\right)}{\epsilon_{\mathbf{k}^{\prime}+\mathbf{q}}-\omega_{i}-E_{1 s, 2 p}+i \Gamma}\right] \\
& \times-\frac{1}{\pi} \operatorname{Im}\left\{\frac{1}{\epsilon_{\mathbf{k}}-\epsilon_{\mathbf{k}+\mathbf{q}}+\boldsymbol{\Omega}+i \gamma_{e}} D_{\nu}(\mathbf{q}, \Omega) \frac{1}{\epsilon_{\mathbf{k}^{\prime}}-\epsilon_{\mathbf{k}^{\prime}+\mathbf{q}}+\Omega-i \gamma_{e}}\right\} .
\end{aligned}
$$



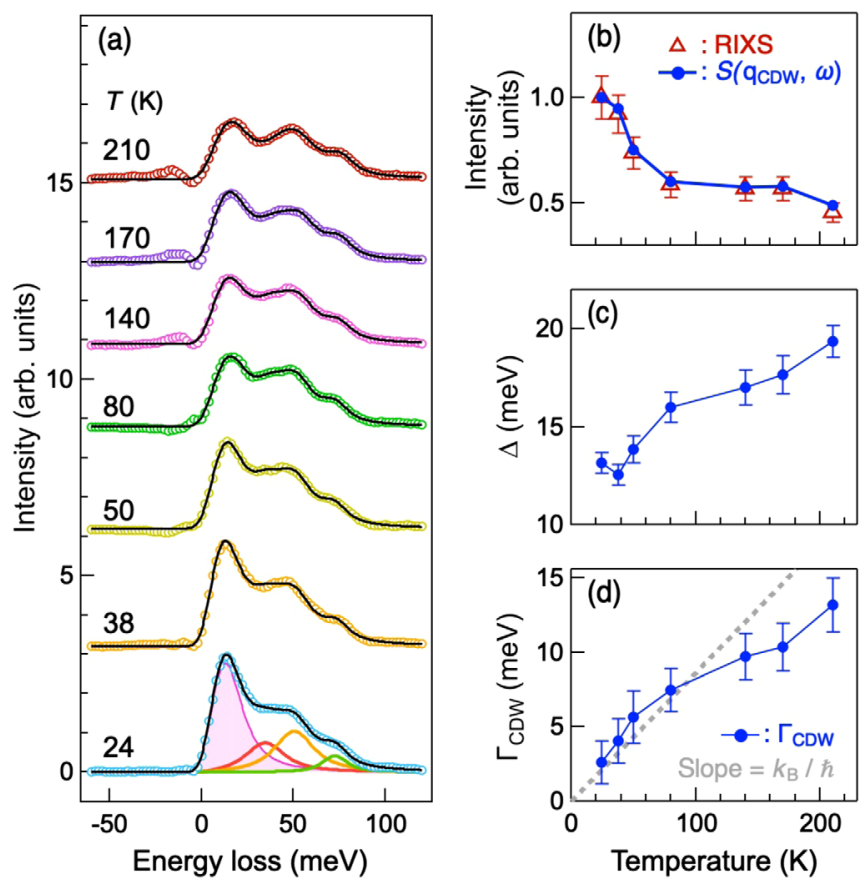

FIG. 3. Temperature dependence of CDW fluctuations. (a) Momentum-integrated RIXS spectra after the subtraction of elastic scattering and then momentum integration over a range from 0.22 to 0.25 in units of $2 \pi / a$ at different temperatures. The RIXS spectra are fitted to four phonon modes. The component derived from the coupling of CDW fluctuations to acoustic phonons is shaded in pink. The components of the BS, $A_{1 g}$, and $B_{1 g}$ phonon modes are shown in green, orange, and red, respectively. Details of the curve fitting are presented in Supplemental Material [47]. (b) RIXS peak intensity of the 14-meV component shown in (a), compared to the maximum of calculated $S\left(\mathbf{q}_{\mathrm{CDW}}, \omega\right)$ after convolution with the instrumental resolution. The value of $S\left(\mathbf{q}_{\mathrm{CDW}}, \omega\right)$ with the 24-K data point is normalized to the RIXS intensity. Values of $\Delta$ and $\Gamma_{\mathrm{CDW}}$ used for the $S\left(\mathbf{q}_{\mathrm{CDW}}, \omega\right)$ calculations are plotted in (c) and (d), respectively. (d) Temperature dependence of $\Gamma_{\mathrm{CDW}}$. The gray dashed line indicates the slope of the lifetime width vs $T$ determined by the limit of the Planckian dissipation. All solid lines connecting data points in (b)-(d) serve as visual guidance.

Here, $n_{f}(x)$ is the Fermi factor; $\epsilon_{\mathbf{k}}$ is the electron band dispersion; $g_{\nu}(\mathbf{k}, \mathbf{q})$ and $D_{\nu}(\mathbf{q}, \xi)$ are the electron-phonon vertex and phonon propagators for phonon branch $\nu$, respectively; $\phi_{2 p_{\alpha}}(\mathbf{k})$ is the oxygen $2 p_{\alpha}$ orbital character of the band; $\hat{\mathbf{r}}_{\alpha}$ is a unit vector pointing along the direction of the oxygen orbital; $\Gamma$ is the core-hole lifetime parameter; $\gamma_{e}$ is a lifetime parameter for the electrons; and $\Omega=$ $\omega_{i}-\omega_{f}$ and $\mathbf{q}=\mathbf{k}_{i}-\mathbf{k}_{f}$ are the energy and momentum, respectively, transferred to the sample. The phonon softening is determined by the total electronic polarizability. Our model essentially assumes that the phonons respond to some strong CDW fluctuation caused by an external source like correlations. That is, the coupling of CDW fluctuations to phonons renormalizes the phonon propagator through charge susceptibility $\chi_{\mathrm{CDW}}\left(\mathbf{q}_{\|}, \omega\right)$. See Supplemental Material [47] for the calculation details.
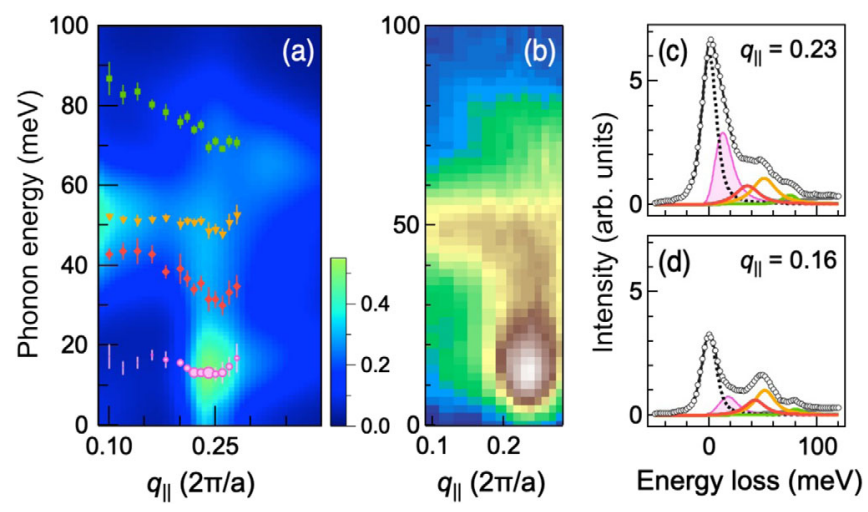

FIG. 4. Phonon excitations from RIXS measurements of optimally doped LSCO. (a) Calculated intensity distribution map using the diagrammatic framework in comparison with phonon dispersions obtained from RIXS spectra of $24 \mathrm{~K}$. Green squares, orange triangles, and red diamonds depict the dispersions of $\mathrm{BS}, A_{1 g}$, and $B_{1 g}$ phonon modes, respectively. The dispersions of the low-energy RIXS excitations are represented by pink circles scaled to the fitted intensity. (b) RIXS intensity distribution map of $T=24 \mathrm{~K}$ extracted from Fig. 2(b) and plotted with the same color scale. (c),(d) Measured RIXS spectra with fitted components for phonon excitations of momenta $q_{\|}=$ 0.23 and 0.16 at $T=24 \mathrm{~K}$, respectively. The curve fitting scheme is the same as that in Fig. 3(a). RIXS data are plotted as open circles with black curves to show the summation of fitted components and a linear background. The component derived from the coupling of CDW fluctuations to acoustic and optical phonons is shaded in pink. The components of the BS, $A_{1 g}$, and $B_{1 g}$ phonon modes are shown in green, orange, and red, respectively. The elastic component is plotted as a dashed curve.

Figure 4(a) presents the calculated RIXS intensity distribution map. That the coupling of CDW fluctuations to acoustic phonons enhances the RIXS intensity significantly at $14 \mathrm{meV}$ for momentum about $\mathbf{q}_{\mathrm{CDW}}$ agrees satisfactorily well with the measurements shown in Fig. 4(b), corroborating the phenomenological form of charge susceptibility given in Eq. (2).

\section{ELECTRON-PHONON COUPLING}

In addition to the pronounced low-energy feature, RIXS also measures other phonon excitations [55,56]. The electron-phonon coupling involved with states near the Fermi level is weak for the apical mode [57]. The contribution of apical phonons to RIXS can be neglected. We, therefore, fit RIXS spectra with three components for highenergy phonons, i.e., bond-stretching (BS), $A_{1 g}$ buckling, and $B_{1 g}$ buckling phonons [30,32,58-60], as shown in Figs. 4(c) and 4(d), which plot fitting spectra of $q_{\|}=0.23$ and 0.16 at $24 \mathrm{~K}$, respectively. Results of other $q_{\|}$and temperatures are presented in Figs. S9-S11 in Supplemental Material [47]. Figure 4(a) also depicts the phonon dispersions from curve fitting. The diagrammatic 
calculations explain well the observed photon softening. In addition to phonon softening, the phonon energies from RIXS agree with those are from density-functional-theory (DFT) calculations [30] and measurements of Raman scattering [59], inelastic neutron scattering [58], IXS [28], and photoemission [60]. We also fit the RIXS data of $T=24 \mathrm{~K}$ with three phonon modes, i.e., BS, buckling, and acoustic phonons; the fitting results plotted in supplemental Fig. S12 [47] show a phonon softening consistent with that from the four-phonon fitting. That is, our curve fitting results show that these phonons all exhibit the strongest softening at $\mathbf{q}_{\|}=(0.25,0)$ rather than $\mathbf{q}_{\mathrm{CDW}}$, supporting the scenario of discommensurate CDWs in LSCO at doping level $x=0.15$. In domains of discommensurate CDWs, phonons are strongly coupled to charge fluctuations, and the phase shift between scattered $\mathrm{x}$ rays from separate domains is negligible. The phonon softening, therefore, occurs at $\mathbf{q}_{\|}=(0.25,0)$ for CDW domains of period $4 a$, in which $a$ is the in-plane lattice constant.

To further understand the characteristics of CDWs in LSCO, we examine the coupling of CDWs to phonons through performing DFT calculations in the scheme of frozen phonons, which are adopted to study qualitatively the coupling of longitudinal BS mode and apical phonon mode of $A_{1 g}$ symmetry with a periodicity of $4 a$. See Supplemental Material [47] for the details. The calculations show that the apical mode has an energy lower than that of the BS mode. Whereas the vibration of either the apical or the BS mode raises the total energy, the vibrations of these two modes together produce an energy gain for the CDW state as compared with the vibration of each single mode. This energy gain indicates that the coupling of these two phonon modes with CDWs is favorable; the coupling strength, measured by the energy gain, is found to be stronger than that with other periodicities, such as $6 a$. The coupling strength also enhances with increasing hole concentrations. These findings are in agreement with the RIXS results that strong phonon softening occurs about $q_{\|}=0.25$. One plausible scenario is that the CDW has effectively a period $4 a$ and is short ranged [51]; a shift in the wave vector of a large sample appears in the Fourier space, giving rise to the formation of discommensurate CDWs.

\section{DISCUSSION AND CONCLUSION}

Although the data presented in the present work are only from a sample near the optimal doping, quantum critical scalings of charge fluctuations in the temperature domain are clearly demonstrated. These results are consistent with the speculation on the existence of a QCP. On the other hand, by suppressing superconductivity in high magnetic fields, linear scalings in resistivity are observed in a more extended regime around the putative QCP near a doping level of 0.2 [39]. While the extended linear-resistivity region shows quantum scaling in temperature, it does not exhibit the quantum critical scaling in the nonthermal parameter, doping. Thus, the correspondence between quantum criticality and $T$-linear resistivity may not be of necessity [61,62]. The apparent extended critical region that is overlapped with the putative QCP may just be a nonFermi-liquid phase [63]. In this scenario, the CDW phase becomes a non-Fermi-liquid phase as the doping level is tuned across the putative QCP. Clearly, to clarify these issues, future work on samples (both LSCO and Nd-LSCO) with different dopings around the critical point and inside the region of long-range charge-ordered phase (Nd-LSCO sample) is required in order to unequivocally prove the existence of QCP related to charge order in LSCO.

In conclusion, we observe short-range CDWs in LSCO near the optimal doping over a wide temperature range; these orders compete locally with the superconducting order. Through combining high-resolution RIXS measurements, theoretical modeling, and diagrammatic calculations, our results provide evidence for phonon softening in superconducting cuprates induced by charge order. The observation of phonon softening indeed results from quantum fluctuations of the charge order.

\section{ACKNOWLEDGMENTS}

We thank Wei-Sheng Lee and Alfred Baron for useful discussions. We thank Peter Towei Huang for making graphic illustrations. This work was supported in part by the Ministry of Science and Technology of Taiwan under Grants No. 109-2112-M-213-010-MY3 and No. 109-2923M-213-001. S. J. acknowledges support from the National Science Foundation under Grant No. DMR-1842056. A. F. K. acknowledges support from the National Science Foundation under Grant No. DMR-1752713. We also thank the support by KAKENHI Grant No. 19K03741 from JSPS and Program for Promoting Researches on the Supercomputer Fugaku (Basic Science for Emergence and Functionality in Quantum Matter JPMXP1020200104) from MEXT.

[1] B. Keimer, S. A. Kivelson, M. R. Norman, S. Uchida, and J. Zaanen, From Quantum Matter to High-Temperature Superconductivity in Copper Oxides, Nature (London) 518, 179 (2015).

[2] E. Fradkin, S. A. Kivelson, and J. M. Tranquada, Colloquium: Theory of Intertwined Orders in High Temperature Superconductors, Rev. Mod. Phys. 87, 457 (2015).

[3] G. Ghiringhelli, M. Le Tacon, M. Minola, S. BlancoCanosa, C. Mazzoli, N.B. Brookes, G. M. De Luca, A. Frano, D. G. Hawthorn, F. He, T. Loew, M. M. Sala, D. C. Peets, M. Salluzzo, E. Schierle, R. Sutarto, G. A. Sawatzky, E. Weschke, B. Keimer, and L. Braicovich, Long-Range Incommensurate Charge Fluctuations in $(Y, \mathrm{Nd}) \mathrm{Ba}_{2} \mathrm{Cu}_{3} \mathrm{O}_{6+x}$, Science 337, 821 (2012). 
[4] E. Blackburn, J. Chang, M. Hücker, A. T. Holmes, N. B. Christensen, R. Liang, D. A. Bonn, W. N. Hardy, U. Rütt, O. Gutowski, M. v. Zimmermann, E. M. Forgan, and S. M. Hayden, X-Ray Diffraction Observations of a Charge-Density-Wave Order in Superconducting Ortho-II $\mathrm{YBa}_{2} \mathrm{Cu}_{3} \mathrm{O}_{6.54}$ Single Crystals in Zero Magnetic Field, Phys. Rev. Lett. 110, 137004 (2013).

[5] T. P. Croft, C. Lester, M. S. Senn, A. Bombardi, and S. M. Hayden, Charge Density Wave Fluctuations in $\mathrm{La}_{2-x} \mathrm{Sr}_{x} \mathrm{CuO}_{4}$ and Their Competition with Superconductivity, Phys. Rev. B 89, 224513 (2014).

[6] X. Zhu, Y. Cao, J. Zhang, E. W. Plummer, and J. Guo, Classification of Charge Density Waves Based on Their Nature, Proc. Natl. Acad. Sci. U.S.A. 112, 2367 (2015).

[7] R. Comin and A. Damascelli, Resonant X-Ray Scattering Studies of Charge Order in Cuprates, Annu. Rev. Condens. Matter Phys. 7, 369 (2016).

[8] H. Miao, R. Fumagalli, M. Rossi, J. Lorenzana, G. Seibold, F. Yakhou-Harris, K. Kummer, N. B. Brookes, G. D. Gu, L. Braicovich, G. Ghiringhelli, and M. P. M. Dean, Formation of Incommensurate Charge Density Waves in Cuprates, Phys. Rev. X 9, 031042 (2019).

[9] J.-J. Wen, H. Huang, S.-J. Lee, H. Jang, J. Knight, Y. S. Lee, M. Fujita, K. M. Suzuki, S. Asano, S. A. Kivelson, C.-C. Kao, and J.-S. Lee, Observation of Two Types of ChargeDensity-Wave Orders in Superconducting $\mathrm{La}_{2-x} \mathrm{Sr}_{x} \mathrm{CuO}_{4}$, Nat. Commun. 10, 3269 (2019).

[10] R. Arpaia, S. Caprara, R. Fumagalli, G. De Vecchi, Y. Y. Peng, E. Andersson, D. Betto, G. M. De Luca, N. B. Brookes, F. Lombardi, M. Salluzzo, L. Braicovich, C. Di Castro, M. Grilli, and G. Ghiringhelli, Dynamical Charge Density Fluctuations Pervading the Phase Diagram of a Cu-Based High-Tc Superconductor, Science 365, 906 (2019).

[11] M. Mitrano, S. Lee, A. A. Husain, L. Delacretaz, M. Zhu, G. de la Peña Munoz, S.X.-L. Sun, Y. I. Joe, A. H. Reid, S. F. Wandel, G. Coslovich, W. Schlotter, T. van Driel, J. Schneeloch, G. D. Gu, S. Hartnoll, N. Goldenfeld, and P. Abbamonte, Ultrafast Time-Resolved X-Ray Scattering Reveals Diffusive Charge Order Dynamics in $\mathrm{La}_{2-x} \mathrm{Ba}_{x} \mathrm{CuO}_{4}$, Sci. Adv. 5, eaax3346 (2019).

[12] D. H. Torchinsky, F. Mahmood, A. T. Bollinger, I. Božović, and N. Gedik, Fluctuating Charge-Density Waves in a Cuprate Superconductor, Nat. Mater. 12, 387 (2013).

[13] R. Comin, A. Frano, M. M. Yee, Y. Yoshida, H. Eisaki, E. Schierle, E. Weschke, R. Sutarto, F. He, A. Soumyanarayanan, Y. He, M. Le Tacon, I. S. Elfimov, J. E. Hoffman, G. A. Sawatzky, B. Keimer, and A. Damascelli, Charge Order Driven by Fermi-Arc Instability in $\mathrm{Bi}_{2} \mathrm{Sr}_{2-x} \mathrm{La}_{x} \mathrm{CuO}_{6+\delta}$, Science 343, 390 (2014).

[14] W. Tabis, Y. Li, M. Le Tacon, L. Braicovich, A. Kreyssig, M. Minola, G. Dellea, E. Weschke, M. J. Veit, M. Ramazanoglu, A. I. Goldman, T. Schmitt, G. Ghiringhelli, N. Barišić, M. K. Chan, C. J. Dorow, G. Yu, X. Zhao, B. Keimer, and M. Greven, Charge Order and Its Connection with FermiLiquid Charge Transport in a Pristine High-Tc Cuprate, Nat. Commun. 5, 5875 (2014).

[15] E. H. da Silva Neto, R. Comin, F. He, R. Sutarto, Y. Jiang, R. L. Greene, G. A. Sawatzky, and A. Damascelli,
Charge Ordering in the Electron-Doped Superconductor $\mathrm{Nd}_{2-x} \mathrm{Ce}_{x} \mathrm{CuO}_{4}$, Science 347, 282 (2015).

[16] G. Campi, A. Bianconi, N. Poccia, G. Bianconi, L. Barba, G. Arrighetti, D. Innocenti, J. Karpinski, N. D. Zhigadlo, S. M. Kazakov, M. Burghammer, M. v. Zimmermann, M. Sprung, and A. Ricci, Inhomogeneity of Charge-DensityWave Order and Quenched Disorder in a High-Tc Superconductor, Nature (London) 525, 359 (2015).

[17] S. Gerber et al., Three-Dimensional Charge Density Wave Order in $\mathrm{YBa}_{2} \mathrm{Cu}_{3} \mathrm{O}_{6.67}$ at High Magnetic Fields, Science 350, 949 (2015).

[18] Y. Y. Peng, R. Fumagalli, Y. Ding, M. Minola, S. Caprara, D. Betto, M. Bluschke, G. M. De Luca, K. Kummer, E. Lefrançois, M. Salluzzo, H. Suzuki, M. Le Tacon, X. J. Zhou, N. B. Brookes, B. Keimer, L. Braicovich, M. Grilli, and G. Ghiringhelli, Re-entrant Charge Order in Overdoped $(\mathrm{Bi}, \mathrm{Pb})_{2.12} \mathrm{Sr}_{1.88} \mathrm{CaCuO}_{6+\delta}$ outside the Pseudogap Regime, Nat. Mater. 17, 697 (2018).

[19] H.-H. Kim, S. M. Souliou, M. E. Barber, E. Lefrançois, M. Minola, M. Tortora, R. Heid, N. Nandi, R. A. Borzi, G. Garbarino, A. Bosak, J. Porras, T. Loew, M. König, P. J. W. Moll, A. P. Mackenzie, B. Keimer, C. W. Hicks, and M. Le Tacon, Uniaxial Pressure Control of Competing Orders in a High-Temperature Superconductor, Science 362, 1040 (2018).

[20] X. M. Chen, C. Mazzoli, Y. Cao, V. Thampy, A. M. Barbour, W. Hu, M. Lu, T. A. Assefa, H. Miao, G. Fabbris, G. D. Gu, J. M. Tranquada, M. P. M. Dean, S. B. Wilkins, and I. K. Robinson, Charge Density Wave Memory in a Cuprate Superconductor, Nat. Commun. 10, 1435 (2019).

[21] B. Yu, W. Tabis, I. Bialo, F. Yakhou, N. B. Brookes, Z. Anderson, Y. Tang, G. Yu, and M. Greven, Unusual Dynamic Charge Correlations in Simple-Tetragonal $\mathrm{HgBa}_{2} \mathrm{Cu}_{2} \mathrm{O}_{4+\delta}$, Phys. Rev. X 10, 021059 (2020).

[22] L. Chaix, G. Ghiringhelli, Y. Y. Peng, M. Hashimoto, B. Moritz, K. Kummer, N. B. Brookes, Y. He, S. Chen, S. Ishida, Y. Yoshida, H. Eisaki, M. Salluzzo, L. Braicovich, Z.X. Shen, T. P. Devereaux, and W. S. Lee, Dispersive Charge Density Wave Excitations in $\mathrm{Bi}_{2} \mathrm{Sr}_{2} \mathrm{CaCu}_{2} \mathrm{O}_{8+\delta}$, Nat. Phys. 13, 952 (2017).

[23] W. S. Lee, K.-J. Zhou, M. Hepting, J. Li, A. Nag, A. C. Walters, M. Garcia-Fernandez, H. C. Robarts, M. Hashimoto, H. Lu, B. Nosarzewski, D. Song, H. Eisaki, Z.X. Shen, B. Moritz, J. Zaanen, and T. P. Devereaux, Spectroscopic Fingerprint of Charge Order Melting Driven by Quantum Fluctuations in a Cuprate, Nat. Phys. 17, 53 (2021).

[24] J. Q. Lin, H. Miao, D. G. Mazzone, G. D. Gu, A. Nag, A. C. Walters, M. García-Fernández, A. Barbour, J. Pelliciari, I. Jarrige, M. Oda, K. Kurosawa, N. Momono, K.-J. Zhou, V. Bisogni, X. Liu, and M. P. M. Dean, Strongly Correlated Charge Density Wave in $\mathrm{La}_{2-x} \mathrm{Sr}_{x} \mathrm{CuO}_{4}$ Evidenced by Doping-Dependent Phonon Anomaly, Phys. Rev. Lett. 124, 207005 (2020).

[25] J. Li, A. Nag, J. Pelliciari, H. Robarts, A. Walters, M. Garcia-Fernandez, H. Eisaki, D. Song, H. Ding, S. Johnston, R. Comin, and K.-J. Zhou, Multiorbital Charge-Density Wave Excitations and Concomitant Phonon Anomalies in $\mathrm{Bi}_{2} \mathrm{Sr}_{2} \mathrm{LaCuO}_{6+\delta}$, Proc. Natl. Acad. Sci. U.S.A. 117, 16219 (2020). 
[26] C. Castellani, C. Di Castro, and M. Grilli, Singular Quasiparticle Scattering in the Proximity of Charge Instabilities, Phys. Rev. Lett. 75, 4650 (1995).

[27] F. Becca, M. Tarquini, M. Grilli, and C. Di Castro, ChargeDensity Waves and Superconductivity as an Alternative to Phase Separation in the Infinite-U Hubbard-Holstein Model, Phys. Rev. B 54, 12443 (1996).

[28] T. Fukuda, J. Mizuki, K. Ikeuchi, K. Yamada, A. Q. R. Baron, and S. Tsutsui, Doping Dependence of Softening in the Bond-Stretching Phonon Mode of $\mathrm{La}_{2-x} \mathrm{Sr}_{x} \mathrm{CuO}_{4}$ $(0 \leq x \leq 0.29)$, Phys. Rev. B 71, 060501(R) (2005).

[29] D. Reznik, L. Pintschovius, M. Ito, S. Iikubo, M. Sato, H. Goka, M. Fujita, K. Yamada, G. D. Gu, and J. M. Tranquada, Electron-Phonon Coupling Reflecting Dynamic Charge Inhomogeneity in Copper Oxide Superconductors, Nature (London) 440, 1170 (2006).

[30] F. Giustino, M. L. Cohen, and S. G. Louie, Small Phonon Contribution to the Photoemission Kink in the Copper Oxide Superconductors, Nature (London) 452, 975 (2008).

[31] M. Le Tacon, A. Bosak, S. M. Souliou, G. Dellea, T. Loew, R. Heid, K.-P. Bohnen, G. Ghiringhelli, M. Krisch, and B. Keimer, Inelastic X-Ray Scattering in $\mathrm{YBa}_{2} \mathrm{Cu}_{3} \mathrm{O}_{6.6}$ Reveals Giant Phonon Anomalies and Elastic Central Peak due to Charge-Density-Wave Formation, Nat. Phys. 10, 52 (2014).

[32] S. R. Park, T. Fukuda, A. Hamann, D. Lamago, L. Pintschovius, M. Fujita, K. Yamada, and D. Reznik, Evidence for a Charge Collective Mode Associated with Superconductivity in Copper Oxides from Neutron and X-Ray Scattering Measurements of $\mathrm{La}_{2-x} \mathrm{Sr}_{x} \mathrm{CuO}_{4}$, Phys. Rev. B 89, 020506(R) (2014).

[33] H. Miao, G. Fabbris, R. J. Koch, D. G. Mazzone, C. S. Nelson, R. Acevedo-Esteves, G. D. Gu, Y. Li, T. Yilimaz, K. Kaznatcheev, E. Vescovo, M. Oda, T. Kurosawa, N. Momono, T. Assefa, I. K. Robinson, E. S. Bozin, J. M. Tranquada, P. D. Johnson, and M. P. M. Dean, Charge Density Waves in Cuprate Superconductors beyond the Critical Doping, npj Quantum Mater. 6, 31 (2021).

[34] S. Sachdev, Where Is the Quantum Critical Point in the Cuprate Superconductors?, Phys. Status Solidi (b) 247, 537 (2010).

[35] S. Sachdev, Quantum Phase Transitions, 2nd ed. (Cambridge University Press, Cambridge, Englandm 2011).

[36] S. Caprara, C. Di Castro, G. Seibold, and M. Grilli, Dynamical Charge Density Waves Rule the Phase Diagram of Cuprates, Phys. Rev. B 95, 224511 (2017).

[37] C. Proust and L. Taillefer, The Remarkable Underlying Ground States of Cuprate Superconductors, Annu. Rev. Condens. Matter Phys. 10, 409 (2019).

[38] B. Michon, C. Girod, S. Badoux, J. Kačmarčík, Q. Ma, M. Dragomir, H. A. Dabkowska, B. D. Gaulin, J.-S. Zhou, S. Pyon, T. Takayama, H. Takagi, S. Verret, N. DoironLeyraud, C. Marcenat, L. Taillefer, and T. Klein, Thermodynamic Signatures of Quantum Criticality in Cuprate Superconductors, Nature (London) 567, 218 (2019).

[39] R. Cooper, Y. Wang, B. Vignolle, O. Lipscombe, S. Hayden, Y. Tanabe, T. Adachi, Y. Koike, M. Nohara, H. Takagi, C. Proust, and N.E. Hussey, Anomalous Criticality in the Electrical Resistivity of $\mathrm{La}_{2-x} \mathrm{Sr}_{x} \mathrm{CuO}_{4}$, Science 323, 603 (2009).
[40] A. Legros, S. Benhabib, W. Tabis, F. Laliberté, M. Dion, M. Lizaire, B. Vignolle, D. Vignolles, H. Raffy, Z. Z. Li, P. Auban-Senzier, N. Doiron-Leyraud, P. Fournier, D. Colson, L. Taillefer, and C. Proust, Universal T-Linear Resistivity and Planckian Dissipation in Overdoped Cuprates, Nat. Phys. 15, 142 (2019).

[41] L. J. P. Ament, M. van Veenendaal, T. P. Devereaux, J. P. Hill, and J. van den Brink, Resonant Inelastic X-Ray Scattering Studies of Elementary Excitations, Rev. Mod. Phys. 83, 705 (2011).

[42] C. Jia, K. Wohlfeld, Y. Wang, B. Moritz, and T. P. Devereaux, Using RIXS to Uncover Elementary Charge and Spin Excitations, Phys. Rev. X 6, 021020 (2016).

[43] S. Komiya, Y. Ando, X. F. Sun, and A. N. Lavrov, c-Axis Transport and Resistivity Anisotropy of Lightly to Moderately Doped $\mathrm{La}_{2-x} \mathrm{Sr}_{x} \mathrm{CuO}_{4}$ Single Crystals: Implications on the Charge Transport Mechanism, Phys. Rev. B 65, 214535 (2002).

[44] S. Komiya, H.-D. Chen, S.-C. Zhang, and Y. Ando, Magic Doping Fractions for High-Temperature Superconductors, Phys. Rev. Lett. 94, 207004 (2005).

[45] S. Ono, S. Komiya, and Y. Ando, Strong Charge Fluctuations Manifested in the High-Temperature Hall Coefficient of High- $T_{c}$ Cuprates, Phys. Rev. B 75, 024515 (2007).

[46] A. Singh et al., Development of the Soft X-Ray AGM-AGS RIXS Beamline at the Taiwan Photon Source, J. Synchrotron Radiat. 28, 977 (2021).

[47] See Supplemental Material at http://link.aps.org/ supplemental/10.1103/PhysRevX.11.041038 for sample characterizations, RIXS setup, calculations, and data analysis.

[48] W. L. McMillan, Theory of Discommensurations and the Commensurate-Incommensurate Charge-Density-Wave Phase Transition, Phys. Rev. B 14, 1496 (1976).

[49] C. H. Chen, J. M. Gibson, and R. M. Fleming, Direct Observation of Charge-Density-Wave Discommensurations and Dislocations in $2 \mathrm{H}$-TaSe ${ }_{2}$, Phys. Rev. Lett. 47, 723 (1981).

[50] W.-L. Tu and T.-K. Lee, Evolution of Pairing Orders between Pseudogap and Superconducting Phases of Cuprate Superconductors, Sci. Rep. 9, 1719 (2019).

[51] A. Mesaros, K. Fujita, S. D. Edkins, M. H. Hamidian, H. Eisaki, S.-i. Uchida, J. C. S. Davis, M. J. Lawler, and E.-A. Kim, Commensurate $4 a_{0}$-Period Charge Density Modulations throughout the $\mathrm{Bi}_{2} \mathrm{Sr}_{2} \mathrm{CaCu}_{2} \mathrm{O}_{8+x}$ Pseudogap Regime, Proc. Natl. Acad. Sci. U.S.A. 113, 12661 (2016).

[52] J. Zaanen, Why the Temperature is High, Nature (London) 430, 512 (2004).

[53] S. Chakravarty, B. I. Halperin, and D. R. Nelson, TwoDimensional Quantum Heisenberg Antiferromagnet at Low Temperatures, Phys. Rev. B 39, 2344 (1989).

[54] S. Chakravarty, Quantum Critical Fluctuations, Planckian Dissipation, and Compactification Scale, arXiv:1907 .12163.

[55] T. P. Devereaux, A. M. Shvaika, K. Wu, K. Wohlfeld, C. J. Jia, Y. Wang, B. Moritz, L. Chaix, W.-S. Lee, Z.-X. Shen, G. Ghiringhelli, and L. Braicovich, Directly Characterizing the Relative Strength and Momentum Dependence of 
Electron-Phonon Coupling Using Resonant Inelastic X-Ray Scattering, Phys. Rev. X 6, 041019 (2016).

[56] L. J. P. Ament, M. van Veenendaal, and J. van den Brink, Determining the electron-phonon coupling strength from Resonant Inelastic X-ray Scattering at transition metal L-edges, Europhys. Lett. 95, 27008 (2011).

[57] S. Johnston, F. Vernay, B. Moritz, Z.-X. Shen, N. Nagaosa, J. Zaanen, and T. P. Devereaux, Systematic Study of Electron-Phonon Coupling to Oxygen Modes across the Cuprates, Phys. Rev. B 82, 064513 (2010).

[58] R. J. McQueeney, Y. Petrov, T. Egami, M. Yethiraj, G. Shirane, and Y. Endoh, Anomalous Dispersion of LO Phonons in $\mathrm{La}_{1.85} \mathrm{Sr}_{1.5} \mathrm{CuO}_{4}$ at Low Temperatures, Phys. Rev. Lett. 82, 628 (1999).

[59] S. Sugai, Y. Takayanagi, N. Hayamizu, T. Muroi, R. Shiozaki, J. Nohara, K. Takenaka, and K. Okazaki, Superconducting Pairing and the Pseudogap in the Nematic
Dynamical Stripe Phase of $\mathrm{La}_{2-x} \mathrm{Sr}_{x} \mathrm{CuO}_{4}$, J. Phys. Condens. Matter 25, 475701 (2013).

[60] X. J. Zhou et al., Multiple Bosonic Mode Coupling in the Electron Self-Energy of $\mathrm{La}_{2-x} \mathrm{Sr}_{x} \mathrm{CuO}_{4}$, Phys. Rev. Lett. 95, 117001 (2005).

[61] Y. Ando, S. Komiya, K. Segawa, S. Ono, and Y. Kurita, Electronic Phase Diagram of High- $T_{c}$ Cuprate Superconductors from a Mapping of the In-Plane Resistivity Curvature, Phys. Rev. Lett. 93, 267001 (2004).

[62] P. Phillips and C. Chamon, Breakdown of One-Parameter Scaling in Quantum Critical Scenarios for High-Temperature Copper-Oxide Superconductors, Phys. Rev. Lett. 95, 107002 (2005).

[63] N. Doiron-Leyraud, I. Walker, L. Taillefer, M. Steiner, S. Julian, and G. Lonzarich, Fermi-Liquid Breakdown in the Paramagnetic Phase of a Pure Metal, Nature (London) 425, 595 (2003). 